\title{
Washing solution and centrifugation affect kinematics of cryopreserved boar semen
}

\author{
Areeg M. Almubarak, Woohyeon Kim, Nabeel H. Abdelbagi, Saddah E. Balla, Il-Jeoung Yu and \\ Yubyeol Jeon*
}

Department of Theriogenology and Reproductive Biotechnology, College of Veterinary Medicine and Bio-Safety Research Institute, Jeonbuk National University, Iksan 54596, Korea

Received March 18, 2021

Revised April 23, 2021

Accepted April 29, 2021

\section{*Correspondence \\ Yubyeol Jeon \\ E-mail: ybjeon@jbnu.ac.kr}

ORCID

https://orcid.org/0000-0003-0328-2974

\begin{abstract}
Cryopreservation is a widely-used efficient means of long-term sperm preservation. However, unlike other types of semen, cryopreserved boar semen has reduced fertility and the efforts continue to optimize post-thawing sperm recovery. In this study, we evaluated the effects of various washing solutions (Hulsen solution, labmade DPBS and commercial DPBS) on post-thawing porcine sperm kinematics (CASA system), viability (SYBR-14/PI) and acrosome integrity (PSA/FITC). We also examined the effect of washing-centrifugation on frozen-thawed semen kinematics. The results indicate that type of washing solution and post-thawing centrifugation alters parameters linked to sperm quality (total motility, progressive motility, viability and acrosome integrity). Significantly higher $(p<0.05)$ motility and progressive motility were obtained when cryopreserved semen was processed with Hulsen solution. The postthaw percentage of live and intact acrosomal sperm was significantly higher in group 1 (Hulsen solution) as compared to other groups. Following thawing-centrifugation, the results showed significantly higher motility and progressive motility in group 1 than other groups. However, the latter two DPBS groups did not differ statistically. Taken together, Frozen-thawed spermatozoa motility, acrosome integrity and viability can be affected by the type of washing solution used. Moreover, centrifugation of frozenthawed semen has an unfavorable effect on total motility and progressive motility.
\end{abstract}

Keywords: boar, centrifugation, cryopreservation, freezing-thawing, washing solution

\section{INTRODUCTION}

Cryopreservation is the most effective method for the long-term storage of semen. Furthermore, cryopreservation enhances genetic utilization by facilitating the distribution of desirable genes and control the transmission of certain diseases (Grossfeld et al., 2008). However, the cryopreservation process exposes semen to physical and chemical stress that impairs sperm quality (Hezavehei et al., 2018). Each step of the cryopreservation including dilution, cooling, freezing, and thawing, diminishes the fertilization potential, with approximately $40 \%$ to $50 \%$ of spermatozoa not even surviving the process (Rath et al., 2009; Watson, 2000). In comparison with fresh semen, insemination with frozen-thawed semen results in lower fertility and farrowing rates by $20 \%$ to $30 \%$ (Knox, 2015; Yeste et al., 2017). Boar spermatozoa in particular are very susceptible to peroxidative damage because of their high polyunsaturated fatty acid content and low concentrations of cholesterol in their plasma membrane (Mandal 
et al., 2014; Yeste, 2015). Although the commercial use of cryopreserved semen for artificial insemination (AI) has become common for many farm animal species worldwide, cryopreserved boar semen has not been integrated into AI centers at a rate comparable to that of other species (DeJarnette et al., 2004; Rodriguez-Martinez and Wallgren, 2010).

There have been numerous attempts to reduce the detrimental functional and structural alterations the cryopreservation process induces in sperm cells. Factors like quality of fresh semen (Parrilla et al., 2012), type and the composition of extender (Park et al., 2012; Blanch et al., 2014), antioxidants supplementation, type and concentration of cryoprotectant (Buranaamnuay et al., 2011; Malo et al., 2010b), and the freezing and thawing rate (Eriksson and Rodriguez-Martinez, 2000) have been tested to improve the freezing-thawing process. Furthermore, different diluents (Noguchi et al., 2015) and centrifugation regimes (Martinez-Alborcia et al., 2012) have been shown to influence post-thaw sperm survival. However, considering the sensitivity of boar semen, the results are still sub-optimal. In this regard, the present study aimed to examine the effects of different washing solutions during cryopreservation of boar semen on post-thawing motility, progressive motility, viability and acrosome integrity. Also, the effect of washing-centrifugation on post-thawing sperm kinematics was evaluated.

\section{MATERIALS AND METHODS}

\section{Chemicals}

Unless otherwise indicated, all chemicals were purchased from Sigma-Aldrich (St. Louis, MO, USA), and prepared using ultra-pure water (ProGen, Genetrone Biotech, Korea). Bovine serum albumin (BSA, REF 10738328) was from Roche Diagnostics (New Zealand). Commercial DPBS was purchased from BYLABS (Lu Science, Korea). Equex-Paste STM was obtained from bestandteil von TGVerdünner für Eber und Hund (Minitube, Germany).

\section{Compositions of washing solutions and freezing extenders}

The components of washing solution used in the present study were shown in Table 1. Hulsen solution (Group 1) was composed of $\mathrm{D}(+)$ Glucose $57.5 \mathrm{~g} / \mathrm{L}, \alpha$-lactose $2.5 \mathrm{~g} / \mathrm{L}$, Sodium citrate $4.5 \mathrm{~g} / \mathrm{L}, \mathrm{Na}_{2}$-EDTA $3.5 \mathrm{~g} / \mathrm{L}, \mathrm{NaHCO}_{3} 1.2 \mathrm{~g} /$
L, KCl $0.4 \mathrm{~g} / \mathrm{L}$ and Gentamycin Sulfate $0.02 \mathrm{~g} / \mathrm{L}$. Lab-made DPBS (Group 2) was composed of $8 \mathrm{~g} / \mathrm{L} \mathrm{NaCl}, 0.2 \mathrm{~g} / \mathrm{L} \mathrm{KCl}$, $0.2 \mathrm{~g} / \mathrm{L} \mathrm{KH}_{2} \mathrm{PO}_{4}, 1.174 \mathrm{~g} / \mathrm{L} \mathrm{Na}_{2} \mathrm{HPO}_{4}, 1 \mathrm{~g} / \mathrm{L} \mathrm{D}(+)$ Glucose, $0.036 \mathrm{~g} / \mathrm{L} \mathrm{Na}$-pyruvate and $1 \mathrm{~g} / \mathrm{L}$ BSA. Commercial DPBS (Group 3) consists of $8.12 \mathrm{~g} / \mathrm{L} \mathrm{NaCl}, 0.20 \mathrm{~g} / \mathrm{L} \mathrm{KCl}, 1.44 \mathrm{~g} /$ $\mathrm{L} \mathrm{Na}_{2} \mathrm{HPO}_{4}$, and $0.20 \mathrm{~g} / \mathrm{L} \mathrm{KH}_{2} \mathrm{PO}_{4}$.

The freezing extenders used in the experiments were extender (1) Tris-egg yolk based extender (TEY), which was composed of TES $12 \mathrm{~g} / \mathrm{L}$, Trizma Base $2 \mathrm{~g} / \mathrm{L}, \mathrm{D}(+)$ Glucose 32 g/L, OEP (Equex) 0.5\% (v/v), Gentamycin Sulfate $0.02 \mathrm{~g} / \mathrm{L}$ and $20 \%$ (v/v) egg yolk. Extender (2) was composed of extender 1 plus $2 \%$ (v/v) glycerol.

\section{Semen collection and transportation}

Porcine semen used in the current study was from duroc boars of proven fertility belonged to a local livestock center (KPG, Korea). Samples were collected by the glovedhand method and were diluted $\left(2.5 \times 10^{9} \pm 0.5\right.$ spermatozoa $/ 90 \mathrm{~mL}$ ) in Beltsville thawing solution (BTS). The diluted semen samples were cooled and maintained at $17^{\circ} \mathrm{C}$ for shipment to the laboratory within $1 \mathrm{~h}$.

\section{Semen cryopreservation}

Spermatozoa were cryopreserved using a modified twostep freezing protocol described by (Córdova et al., 1997). Semen was analyzed upon arrival to ensure its quality and only samples with greater than $80 \%$ motility were used in this study. The diluted semen was transferred to a $15 \mathrm{~mL}$ conical tube and equilibrated at $17^{\circ} \mathrm{C}$. After $2 \mathrm{~h}$ semen was centrifuged at $2,000 \mathrm{rpm}$ for $5 \mathrm{~min}$ at $15^{\circ} \mathrm{C}$, whereupon the supernatant was discarded and the pellet

Table 1. Composition of various washing solutions

\begin{tabular}{|c|c|c|c|}
\hline Component (g/L) & $\begin{array}{l}\text { Hulsen } \\
\text { solution }\end{array}$ & Lab DPBS & $\begin{array}{c}\text { Commercial } \\
\text { DPBS }\end{array}$ \\
\hline $\mathrm{D}(+)$ Glucose & 57.50 & 1.00 & - \\
\hline$\alpha$-lactose & 2.50 & - & - \\
\hline Sodium citrate $\cdot 2 \mathrm{H}_{2} \mathrm{O}$ & 4.50 & - & - \\
\hline $\mathrm{Na}_{2}$ EDTA & 3.50 & - & - \\
\hline $\mathrm{NaHCO}_{3}$ & 1.20 & - & - \\
\hline $\mathrm{KCl}$ & 0.40 & 0.20 & 0.20 \\
\hline Gentamycin Sulfate & 0.02 & - & - \\
\hline $\mathrm{NaCl}$ & - & 8.00 & 8.12 \\
\hline $\mathrm{KH}_{2} \mathrm{PO}_{4}$ & - & 0.20 & 0.20 \\
\hline $\mathrm{Na}_{2} \mathrm{HPO}_{4}$ & - & 1.174 & 1.44 \\
\hline $\mathrm{Na}$-pyruvate & - & 0.036 & - \\
\hline BSA & - & 1.00 & - \\
\hline
\end{tabular}


was resuspended in each washing solution according to experimental design. Each solution was then centrifuged at $2,000 \mathrm{rpm}$ for $5 \mathrm{~min}$ at $15^{\circ} \mathrm{C}$. The supernatant was then discarded and the sperm pellet $\left(2 \times 10^{8}\right.$ spermatozoa $\left./ \mathrm{mL}\right)$ was resuspended in extender 1 . The extended semen was cooled at $5^{\circ} \mathrm{C}$ for $60 \mathrm{~min}$, after which the cooled-extended semen was mixed 1:1 (v/v) with extender 2 . The extended semen was then loaded into $0.5 \mathrm{~mL}$ straws (Fujihira, Japan), sealed and incubated at $5^{\circ} \mathrm{C}$ for $20 \mathrm{~min}$. Straws were placed in a polystyrene box $4 \mathrm{~cm}$ above liquid nitrogen vapor for $20 \mathrm{~min}$ before being plunged into liquid nitrogen for storage.

\section{Assessment of frozen-thawed semen kinematics}

The frozen semen was thawed in a water bath at $38^{\circ} \mathrm{C}$ for $25 \mathrm{sec}$. Then, diluted according to the experiment design in each washing solution 1:4 (v/v). Frozen-thawed (FT) sperm motility was measured using computerassisted sperm analysis (Sperm Class Analyzer, Microptic, Spain). Briefly, $5 \mu \mathrm{L}$ of semen was placed in a counting chamber (GoldCyto, Microptic, Spain) on a heated stage $38^{\circ} \mathrm{C}$. For each analysis, three fields were evaluated and at least 1,000 cells were counted. Motility patterns including total sperm motility (TM, \%), progressive motility (PM, \%), rapid progressive motility (RPM, \%) and medium progressive motility (MPM, \%) were measured.

\section{Assessment of sperm viability}

The determination of FT sperm viability was carried out using LIVE/DEAD ${ }^{\circledR}$ Sperm Viability Kit (ThermoFisher, USA) following the method of (Yu and Leibo, 2002). In brief, $5 \mu \mathrm{L}$ SYBR-14 was added to $50 \mu \mathrm{L}$ spermatozoa and incubated for $5 \mathrm{~min}$ in the dark. Then, $5 \mu \mathrm{L}$ PI was added, and the mixture was incubated again for $5 \mathrm{~min}$. Two smears from each group were made using the semen-stain mixture and dried in the air, then examined under a fluorescence microscope (Axio, Carl Zeiss). Appropriately 200 spermatozoa were counted per slide and classified as live (green fluorescent) or dead spermatozoa (red fluorescent).

\section{Assessment of acrosome integrity}

Sperm acrosome integrity was evaluated using the fluorescent stain method described by (Yu and Leibo, 2002). Briefly, thin smears were made from FT semen of each group and dried in the air. Smears were fixed with absolute methanol and stained with Pisum sativum aggluti- nin (PSA) labeled with fluorescein isothiocyanate (FITC). Stained smears were covered with parafilm for $20 \mathrm{~min}$, dipped in distilled water for $15 \mathrm{~min}$ and then allowed to dry. For each replicate, two slides were examined using a fluorescence microscope (Axio, Carl Zeiss). The percentage of acrosome-intact sperm (spermatozoa with strong green fluorescence on acrosomal region) was counted in a minimum of 200 sperms per slide.

\section{Experimental design}

In experiment one, the effect of using different washing solutions during the cryopreservation process was assessed. Spermatozoa were washed (using either Hulsen solution, lab-made DPBS or commercial DPBS), extended in TEY extender, cooled and cryopreserved as described above. After thawing, each sample was diluted with the same washing solution, then sperm motility, progressive motility, viability and acrosome integrity for each group were evaluated. In experiment two, the effect of dilution with each solution and centrifugation on post-thawing sperm parameters were assessed. FT semen was diluted and centrifuged at 2,000 rpm for 2 min (using either Hulsen solution, lab-made DPBS or commercial DPBS), the supernatant was discarded and pellets were resuspended in each washing solution, then motility parameters were assessed as described above using CASA system.

\section{Statistical analyses}

Each experiment was repeated a minimum of four times. Data were analyzed using SAS software, version 9.4 (SAS Institute Inc., Cary, NC, USA). Percentage of data were compared by one-way ANOVA, followed by Duncan' $s$ multiple range test. The results are expressed as mean \pm standard error and values of $p<0.05$ were considered significant.

\section{RESULTS}

Effects of washing solution on post-thawing sperm motility

The percentage of motile spermatozoa and spermatozoa exhibited progressive motility was significantly higher in the group processed with Hulsen solution in comparison to other groups $(p<0.05)$. There was no statistically significant difference in the percentage of FT motile and progressive motile sperm between the two DPBS groups (Fig. 1). 


\section{Effects of washing solution on post-thawing sperm viability}

As shown in Fig. 2, the post-thaw percentage of live sperm was higher $(p<0.05)$ in Group 1 as compared to other groups $(40.25 \pm 1.91,33.03 \pm 4.47$ and $28.10 \pm$ 2.97) for Hulsen solution, lab and commercial DPBS, respectively.

\section{Effects of washing solution on acrosome integrity}

The results of the post-thaw semen analysis shown in Fig. 3, indicated that the percentage of sperm with the

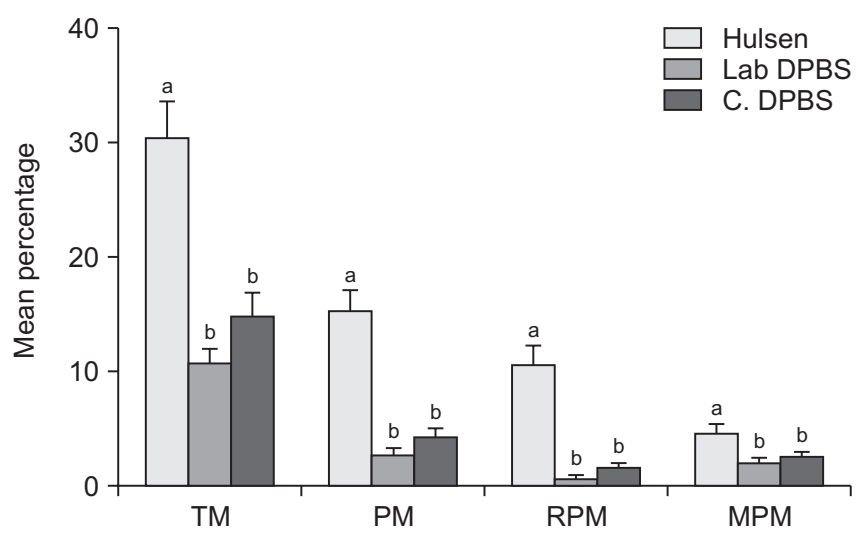

Fig. 1. Effect of different washing solutions used during cryopreservation of boar semen on post-thawing sperm motility. TM: total motility, PM: progressive motility, RPM: rapid progressive motility, MPM: medium progressive motility. Letters a and $b$ represent significant differences between groups $(p<0.05)$. Error bars show the standard error of the mean.

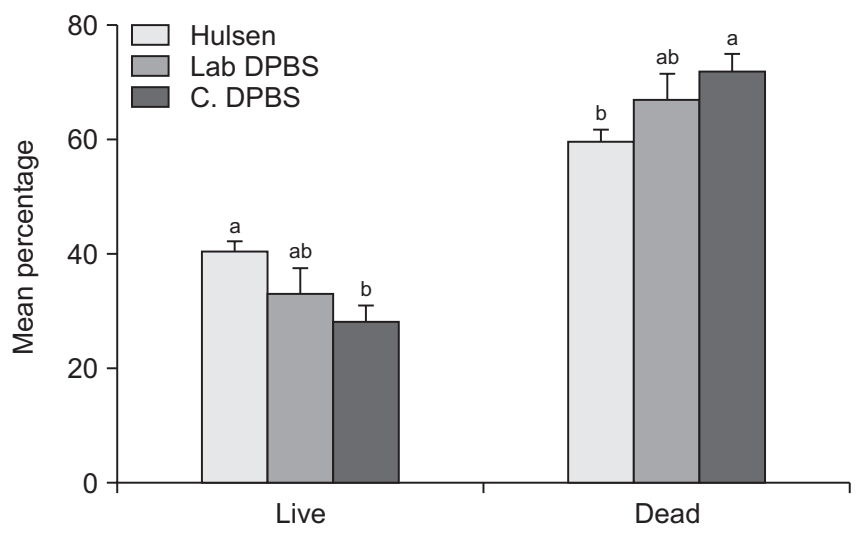

Fig. 2. Effect of different washing solutions used during cryopreservation of boar semen on post-thawing sperm viability. Frozen-thawed spermatozoa were stained with Sybr-14/PI and examined under green and red fluorescence. Stained spermatozoa were classified as live (Sybr-14+) or dead (PI+). Values are expressed as the mean $\pm S E$. Letters $\mathrm{a}$ and $\mathrm{b}$ represent significant differences between groups $(p<0.05)$. Error bars show the standard error of the mean. normal acrosomal region was significantly higher $(p<$ $0.05)$ in group 1 than other groups $(42.50 \% \pm 2.34 \%$, $35.02 \% \pm 1.56 \%$ and $37.18 \% \pm 2.15 \%)$ for Hulsen solution, lab and commercial DPBS, respectively.

\section{Effect of post-thawing centrifugation on sperm kinematics}

The effect of washing-centrifugation on frozen-thawed semen shown in Fig. 4. The percentage of FT motile and progressive motile sperm after centrifugation was higher in Group 1 as compared to other groups $(p<0.05)$. There

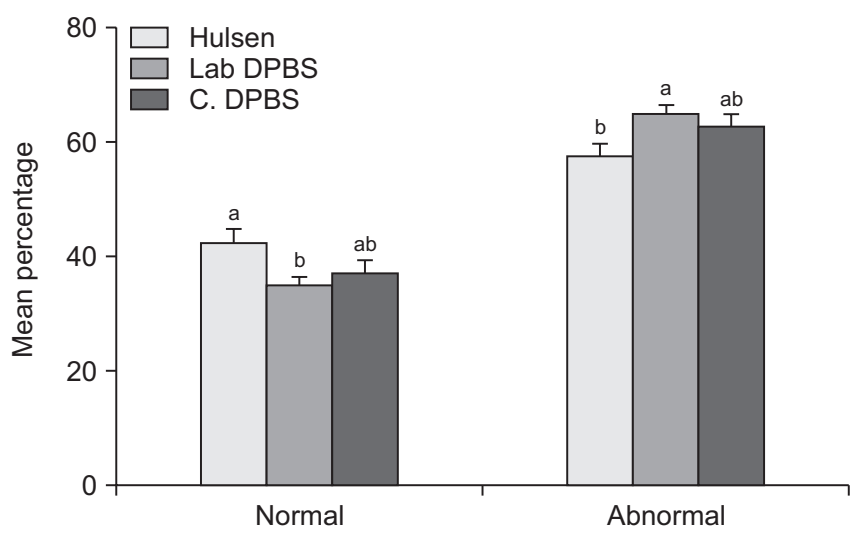

Fig. 3. Effect of different washing solution used during cryopreservation of boar semen on post-thawing acrosome integrity. Frozenthawed spermatozoa were stained with PSA/FITC and examined under green fluorescence. Stained spermatozoa were classified as normal (intact acrosome) or abnormal (un-intact acrosome). Values are expressed as the mean \pm SE. Letters a and b represent significant differences between groups $(p<0.05)$. Error bars show the standard error of the mean.

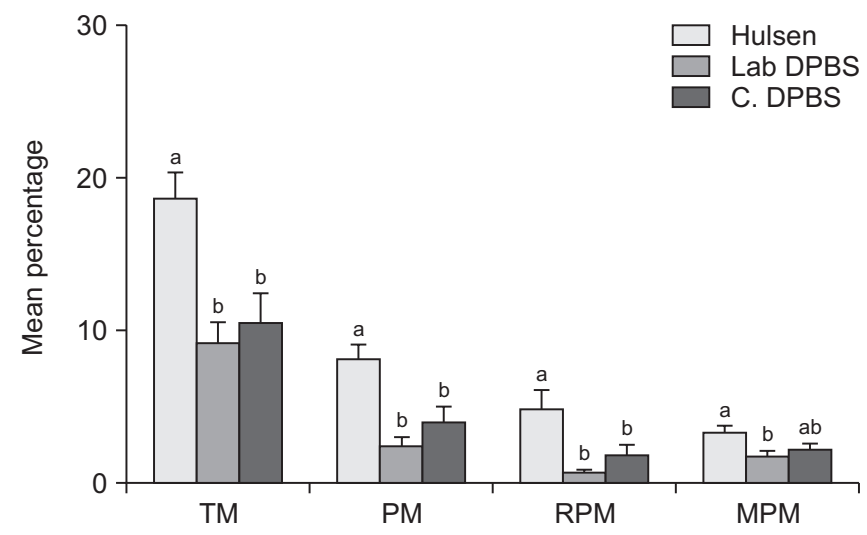

Fig. 4. Effect of post-thawing washing-centrifugation of cryopreserved boar semen on sperm motility. TM: total motility, PM: progressive motility, RPM: rapid progressive motility, MPM: medium progressive motility. Letters a and b represent significant differences between groups $(p<0.05)$. Error bars show the standard error of the mean. 
was no statistically significant difference in the percentage of FT motile and progressive motile sperm between the two other groups.

\section{DISCUSSION}

Cryopreservation is an efficient method for semen storage that allows for long-term preservation and distribution of superior genetic material throughout the animal industry. However, sperm subjected to the cooling-freezing-thawing process has a short lifespan and reduced fertility (Jovičič et al., 2020). It has been shown that this is due to both a loss of sperm viability and impairment of function among survivors (Watson, 2000). Notably, cryopreserved boar sperm, which is particularly sensitive to the cellular stress imposed by cryopreservation, emerges from this process with poor viability (Rath et al., 2009). However, increase understanding of this process may lead to improved cryopreservation methods and methodological refinements that enhance post-thaw viability and fertility.

Considering the aforementioned, more work is required for the cryopreservation protocols to be fully optimized. Several studies have attempted to improve cryopreserved semen quality by supplementing extenders with antioxidants (Luño et al., 2014; Lee and Kim, 2017) and proteins (Park et al., 2018; Robles et al., 2019), and adding saccharides (Pezo et al., 2020). Other researchers have reported that freezing-thawing protocol can affect with diluent type and centrifugation regime (Carvajal et al., 2004; Stuart et al., 2019). In the present study, we determined that the type of washing solution used affects the quality of the frozen-thawed boar semen. Significantly higher motility and progressive motility were obtained when cryopreserved semen was processed with Hulsen solution. Concerning post-thawing sperm viability and acrosome integrity, the best results were also observed in the group processed with Hulsen solution. These parameters are an important indicator of semen quality and have been correlated with fertility (Foote, 2003).

The presence of sugar in the Hulsen solution might explain the better results obtained from this group when compared to other DPBS groups. The exact mechanism by which sugars improve semen's freezability is not fully understood, though it has been suggested that a hypertonic solution of sugars generates osmotic pressure, reducing the amount of intracellular water and intracellular ice formation, thereby preserving the sperm during the freezing process and rendering the membrane less vulnerable to the morphological changes that occur during the rapid reflux of water (Jain and Roy, 2009; Malo et al., 2010a). Furthermore, The beneficial effects of using sugar during semen freezing-thawing process in this study concur with previous reports in the literature that demonstrated glucose (Ciereszko et al., 2014), trehalose (Athurupana et al., 2015), and raffinose plus fructose (Yildiz et al., 2007) delivered high motility and plasma membrane integrity rates after sperm freezing and thawing.

Alternatively, the presence of sodium EDTA as a component of Hulsen solution might have stimulated total and progressive motility in the frozen-thawed boar spermatozoa. It has been reported elsewhere that supplementing sperm preparation medium with EDTA significantly improved forward motility and reduced DNA fragmentation in human sperm (Chi et al., 2008) and buffalo sperm (Hussain et al., 2019).

Centrifugation, which is part of semen freezing-thawing protocol, is essential for the preparation of sperm for further uses in in-vitro fertilization and other assisted reproduction technologies. In the present study, we examined the combined effect of washing-centrifugation of frozenthawed boar semen. Post-thaw semen analysis showed significantly higher motility and progressive motility in Group 1 (Hulsen solution) than other groups. Notably, we also observed lower total motility and progressive motility after centrifugation of FT semen. This finding consistent with that of (Shekarriz et al., 1995), who demonstrated that centrifugation imparts stress to spermatozoa and may increase lipid peroxidation. Previous studies concerning the impact of various centrifugation regimes on boar spermatozoa have reported that the use of shortterm centrifugation with a relatively high force has a positive effect on boar sperm's cryosurvival (Jovičič et al., 2020). Accordingly, the results presented in the literature regarding the washing of spermatozoa are quite variable. Further studies are needed to clarify this issue certainly in terms of post-thawing centrifugation.

\section{CONCLUSION}

In conclusion, the present study demonstrated that the type of washing solution used during the freezing-thawing 
process affects semen quality, FT semen processed with Hulsen solution showed enhanced motion parameters, higher rate of viability and acrosome integrity than other groups. Furthermore, centrifugation of frozen-thawed semen has an unfavorable effect on total motility and progressive motility.

\section{CONFLICTS OF INTEREST}

No potential conflict of interest relevant to this article was reported.

\section{ACKNOWLEDGEMENTS}

This work was supported by the Basic Science Research Program administered through the National Research Foundation of Korea (NRF) and funded by the Ministry of Education (2020R1I1A3071673), and with funds provided by Jeonbuk National University.

\section{AUTHOR CONTRIBUTIONS}

Conceptualization: A. M. Almubarak, W. Kim

Data curation: A. M. Almubarak, W. Kim, Y. Jeon

Formal analysis: A. M. Almubarak, N. H. Abdelbagi

Funding acquisition: I. Yu, Y. Jeon

Investigation: A. M. Almubarak, W. Kim, I. Yu, Y. Jeon

Methodology: A. M. Almubarak, W. Kim. S. E. Balla

Project administration: I. Yu, Y. Jeon

Resources: I. Yu, Y. Jeon

Software: A. M. Almubarak, N. H. Abdelbagi, W. Kim, S. E. Balla

Supervision: I. Yu, Y. Jeon

Validation: I. Yu, Y. Jeon

Visualization: I. Yu, Y. Jeon

Writing - original draft: A. M. Almubarak

Writing - review \& editing: A. M. Almubarak, I. Yu, Y. Jeon

\section{AUTHOR'S POSITION AND ORCID NO.}

AM Almubarak, Ph.D. Student, https://orcid.org/0000-0002-8005-6885

W Kim, B.S. Student, https://orcid.org/0000-0002-0036-5005

NH Abdelbagi, Ph.D. Student, https://orcid.org/0000-0002-0939-6705
SE Balla, Ph.D. Student, https://orcid.org/0000-0002-7382-2828

IJ Yu, Professor, https://orcid.org/0000-0002-5530-5974

Y Jeon, Professor, https://orcid.org/0000-0003-0328-2974

\section{REFERENCES}

Athurupana R, Takahashi D, Ioki S, Funahashi H. 2015. Trehalose in glycerol-free freezing extender enhances post-thaw survival of boar spermatozoa. J. Reprod. Dev. 61:205-210.

Blanch E, Tomás C, Hernández M, Roca J, Martínez EA, Vázquez JM, Mocé E. 2014. Egg yolk and glycerol requirements for freezing boar spermatozoa treated with methyl $\beta$-cyclodextrin or cholesterol-loaded cyclodextrin. J. Reprod. Dev. 60:143-149.

Buranaamnuay K, Grossfeld R, Struckmann C, Rath D. 2011. Influence of cryoprotectants glycerol and amides, combined with antioxidants on quality of frozen-thawed boar sperm. Anim. Reprod. Sci. 127:56-61.

Carvajal G, Cuello C, Ruiz M, Vázquez JM, Martínez EA, Roca J. 2004. Effects of centrifugation before freezing on boar sperm cryosurvival. J. Androl. 25:389-396.

Chi HJ, Kim JH, Ryu CS, Lee JY, Park JS, Chung DY, Choi SY, Kim MH, Chun EK, Roh SI. 2008. Protective effect of antioxidant supplementation in sperm-preparation medium against oxidative stress in human spermatozoa. Hum. Reprod. 23:10231028.

Ciereszko A, Dietrich GJ, Nynca J, Dobosz S, Zalewski T. 2014. Cryopreservation of rainbow trout semen using a glucosemethanol extender. Aquaculture 420-421:275-281.

Córdova A, Ducolomb Y, Jiménez I, Casas E, Bonilla E, Betancourt M. 1997. In vitro fertilizing capacity of frozen-thawed boar semen. Theriogenology 47:1309-1317.

DeJarnette JM, Marshall CE, Lenz RW, Monke DR, Ayars WH, Sattler CG. 2004. Sustaining the fertility of artificially inseminated dairy cattle: the role of the artificial insemination industry. J. Dairy Sci. 87 Suppl:E93-E104.

Eriksson BM and Rodriguez-Martinez H. 2000. Effect of freezing and thawing rates on the post-thaw viability of boar spermatozoa frozen in FlatPacks and Maxi-straws. Anim. Reprod. Sci. 63:205-220.

Foote RH. 2003. Fertility estimation: a review of past experience and future prospects. Anim. Reprod. Sci. 75:119-139.

Grossfeld R, Sieg B, Struckmann C, Frenzel A, Maxwell WM, Rath D. 2008. New aspects of boar semen freezing strategies. Theriogenology 70:1225-1233.

Hezavehei M, Sharafi M, Kouchesfahani HM, Henkel R, Agarwal A, Esmaeili V, Shahverdi A. 2018. Sperm cryopreservation: a review on current molecular cryobiology and advanced approaches. Reprod. Biomed. Online 37:327-339.

Hussain N, Andrabi SMH , Mehmood MU. 2019. Effect of EDTA 
as chelating agent in extender on the post thaw quality of buffalo bull spermatozoa. Cryo Letters 40:159-163.

Jain NK and Roy I. 2009. Effect of trehalose on protein structure. Protein Sci. 18:24-36.

Jovičić M, Chmelíková E, Sedmíková M. 2020. Cryopreservation of boar semen. Czech J. Anim. Sci. 65:115-123.

Knox RV. 2015. The fertility of frozen boar sperm when used for artificial insemination. Reprod. Domest. Anim. 50 Suppl 2:90-97.

Lee EJ and Kim DY. 2017. Effects of curcumin from turmeric supplementation in freezing buffer on sperm motilities and reactive oxygen species generation. J. Emb. Trans. 32:249255.

Luño V, Gil L, Olaciregui M, González N, Jerez RA, de Blas I. 2014. Rosmarinic acid improves function and in vitro fertilising ability of boar sperm after cryopreservation. Cryobiology 69:157-162.

Malo C, Gil L, Gonzalez N, Cano R, de Blas I, Espinosa E. 2010a. Comparing sugar type supplementation for cryopreservation of boar semen in egg yolk based extender. Cryobiology 61:17-21.

Malo C, Gil L, Gonzalez N, Martínez F, Cano R, de Blas I, Espinosa E. 2010b. Anti-oxidant supplementation improves boar sperm characteristics and fertility after cryopreservation: comparison between cysteine and rosemary (Rosmarinus officinalis). Cryobiology 61:142-147.

Mandal R, Badyakar D, Chakrabarty J. 2014. Role of membrane lipid fatty acids in sperm cryopreservation. Adv. Androl. 2014:190542.

Martinez-Alborcia MJ, Morrell JM, Parrilla I, Barranco I, Vázquez JM, Martinez EA, Roca J. 2012. Improvement of boar sperm cryosurvival by using single-layer colloid centrifugation prior freezing. Theriogenology 78:1117-1125.

Noguchi M, Yoshioka K, Hikono H, Suzuki C, Kikuchi K. 2015. Effect of semen extenders on frozen-thawed boar sperm characteristics and distribution in the female genital tract after deep intrauterine insemination in sows. Anim. Reprod. Sci. 163:164-171.

Park CH, Kim SW, Hwang YJ, Kim DY. 2012. Cryopreservation with trehalose reduced sperm chromatin damage in miniature pig. J. Emb. Trans. 27:107-111.

Park SH, Oh KB, Ock SA, Byun SJ, Lee HC, Kumar S, Lee SG, Woo JS. 2018. Effects of ice-binding protein from Leucosporidium on the cryopreservation of boar sperm*. J. Emb.
Trans. 33:185-194.

Parrilla I, del Olmo D, Sijses L, Martinez-Alborcia MJ, Cuello C, Vazquez JM, Martinez EA, Roca J. 2012. Differences in the ability of spermatozoa from individual boar ejaculates to withstand different semen-processing techniques. Anim. Reprod. Sci. 132:66-73.

Pezo F, Zambrano F, Uribe P, Risopatrón J, Moya C, Cesar de Andrade AF, Burgos RA, Yeste M, Sánchez R. 2020. Oxidative and nitrosative stress in frozen-thawed pig spermatozoa. II: effect of the addition of saccharides to freezing medium on sperm function. Cryobiology 97:5-11.

Rath D, Bathgate R, Rodriguez-Martinez H, Roca J, Strzezek J, Waberski D. 2009. Recent advances in boar semen cryopreservation. Soc. Reprod. Fertil. Suppl. 66:51-66.

Robles V, Valcarce DG, Riesco MF. 2019. The use of antifreeze proteins in the cryopreservation of gametes and embryos. Biomolecules 9:181.

Rodriguez-Martinez H and Wallgren M. 2010. Advances in boar semen cryopreservation. Vet. Med. Int. 2010:396181.

Shekarriz M, DeWire DM, Thomas AJ Jr, Agarwal A. 1995. A method of human semen centrifugation to minimize the iatrogenic sperm injuries caused by reactive oxygen species. Eur. Urol. 28:31-35.

Stuart CC, Vaughan JL, Kershaw CM, de Graaf SP, Bathgate R. 2019. Effect of diluent type, cryoprotectant concentration, storage method and freeze/thaw rates on the post-thaw quality and fertility of cryopreserved alpaca spermatozoa. Sci. Rep. 9:12826.

Watson PF. 2000. The causes of reduced fertility with cryopreserved semen. Anim. Reprod. Sci. 60-61:481-492.

Yeste M. 2015. Recent advances in boar sperm cryopreservation: state of the art and current perspectives. Reprod. Domest. Anim. 50 Suppl 2:71-79.

Yeste M, Rodríguez-Gil JE, Bonet S. 2017. Artificial insemination with frozen-thawed boar sperm. Mol. Reprod. Dev. 84:802-813.

Yildiz C, Ottaviani P, Law N, Ayearst R, Liu L, McKerlie C. 2007. Effects of cryopreservation on sperm quality, nuclear DNA integrity, in vitro fertilization, and in vitro embryo development in the mouse. Reproduction 133:585-595.

Yu I and Leibo SP. 2002. Recovery of motile, membrane-intact spermatozoa from canine epididymides stored for 8 days at 4 degrees C. Theriogenology 57:1179-1190. 